\title{
Wenn es im Ohr klingelt
}

BERTHOLD LANGGUTH UND TOBIAS KLEINJUNG

\author{
Ein 48-jähriger Mann stellt sich mit einem akut aufge- \\ tretenen linksseitigen Ohrgeräusch vor. Die Anamnese \\ ergibt darüber hinaus einen linksseitigen Gesichts- \\ schmerz sowie Z. n. HWS-Schleudertrauma durch einen \\ Verkehrsunfall eine Woche zuvor.
}

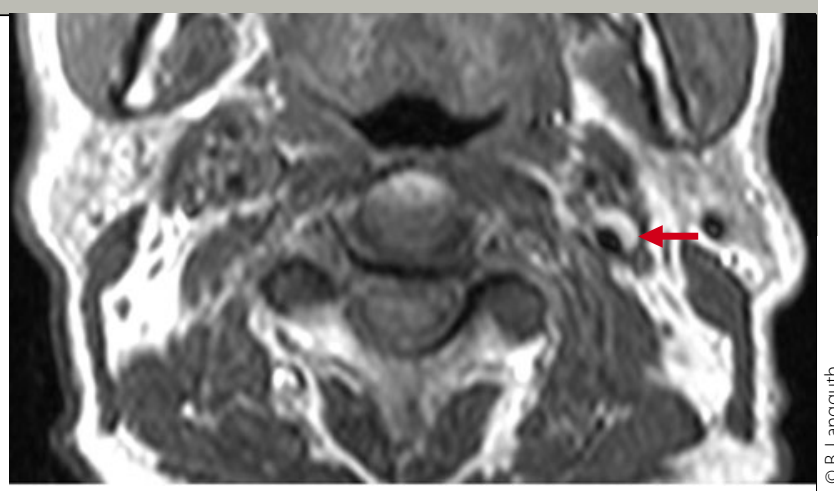

Karotisdissektion links (Pfeil).

\section{Mögliche Ursachen}

Häufig tritt Tinnitus im Zusammenhang mit einem Hörverlust auf. Neben Innenohrschädigungen (z. B. Hörsturz, Altersschwerhörigkeit, Knalltrauma, Nebenwirkungen einer medikamentösen Therapie, Morbus Menière) oder Mittelohrpathologien (z.B. Mittelohrentzündung, Otosklerose) können auch Schädigungen des Hörnerven (mikrovaskuläre Kompression, Akustikusneurinom) dafür verantwortlich sein. Ebenso kann Tinnitus ein Begleitsymptom bei zerebrovaskulären Erkrankungen (z. B. Karotisstenose, -dissektion, arteriovenöser Gefäßmalformation oder Aneurysma) sein.

Ohrgeräusche können mit Kopfschmerzsyndromen (Migräne oder andere trigeminovaskuläre Kopfschmerzsyndrome) einhergehen oder Folge von $\mathrm{Pa}$ thologien im Bereich der HWS und des Kiefergelenkes sein. In seltenen Fällen können zentrale Läsionen ursächlich sein, z. B. ischämische Läsionen im inferioren Colliculus. Auch Begleitsymptome von Tinnitus wie eine akute depressive Symptomatik mit Suizidalität sollten bei der Primärdiagnostik bedacht werden.

\section{Vorgehen bei akutem Tinnitus}

- Von entscheidender Bedeutung für das weitere Vorgehen ist die Anamnese.

- Trat im zeitlichen Zusammenhang mit dem Tinnitus eine Hörminderung auf? Patienten haben manchmal den Eindruck, dass der Tinnitus die Hörstörung bedingt. In Wirklichkeit ist der Kausal- zusammenhang umgekehrt. Im Zweifelsfall ist bei V. a. eine akute Hörstörung immer die weitere HNO-ärztliche Diagnostik inkl. Tonaudiogramm indiziert. Der HNO-Arzt kann dann die weitere Diagnostik und Behandlung einleiten.

- Handelt sich um einen pulssynchronen Tinnitus? Dies kann durch Abgleich der verspürten Tinnituspulsationen und den am Handgelenk gefühlten Pulsschlägen festgestellt werden. Auch eine Auskultation der periaurikulären Region und des Halses kann nützlich sein. Bei pulssynchronem Tinnitus Überweisung zum Neurologen (neurovaskuläre bzw. -radiologische Diagnostik).

- Tritt der Tinnitus episodisch auf? Entsprechend der Begleitsymptome kann er weiter klassifiziert werden. Episodischer Tinnitus mit Schwerhörigkeit, Ohrdruck und Schwindel deuten auf einen $\mathrm{M}$. Menière hin, mit einseitigem Kopfschmerz auf Migräne.

- Tinnitus im Zusammenhang mit einem HWS- bzw. Schädeltrauma erfordert eine spezifische Diagnostik, auch wenn das Trauma mehrere Wochen zurückliegt.

- Nicht selten treten auch psychiatrische Störungen auf. Bei V. a. eine Angststörung oder Depression, insbesondere bei V. a. Suizidalität sollte die psychiatrische Mitbehandlung eingeleitet werden.

- Manche der aufgelisteten Konstellationen sind zwar selten, sollten jedoch im Zweifelsfall ausgeschlossen werden, da sich wichtige therapeutische Konsequenzen daraus ergeben können.
- In der Mehrzahl der Fälle liegt dem Tinnitus keine schwerwiegende Erkrankung zugrunde.

- Wichtig ist, dem Patienten zu vermitteln, dass der Spontanverlauf günstig ist. - Weitere detaillierte Information zur Diagnostik und Therapie von Tinnitus: www. tinnitusresearch.org/en/documents/ downloads/TRI_Tinnitus_Flowchart.pdf

\section{Für die Verfasser:}

Priv.-Doz. Dr. med. Berthold Langguth, Klinik und Poliklinik für Psychiatrie und Psychotherapie der Univ. Regensburg am Bezirksklinikum, Universitätsstr. 84, D-93053 Regensburg, E-Mail: Berthold.Langguth@medbo.de

Koautor: Priv.-Doz. Dr. med. T. Kleinjung, Klinik für HNO-Heilkunde, Universitätsklinik Zürich/Schweiz.

\section{Tabelle 1}

\section{Indikationen für weitere} diagnostische Maßnahmen

- Hörverlust

_ Pulssynchroner pulsatiler Tinnitus

_ Posttraumatischer Tinnitus

_ Akute psychiatrische Komorbidität

\section{Kasuistik}

\section{Wie ging es weiter?}

In der MR-Angiografie fand sich eine Dissektion der linken Arteria carotis interna. Es erfolgte die Heparinisierung über sechs Monate, unter der die Karotisdissektion folgenlos ausheilte. Der Tinnitus blieb jedoch in reduzierter Intensität bestehen. 